\title{
Medial preoptic area knife cuts in the lactating female rat: Effects on maternal behavior and pituitary-adrenal activity
}

\author{
W. P. SMOTHERMAN, J. W. HENNESSY, and S. LEVINE \\ Department of Psychiatry and Behavioral Sciences \\ Stanford University School of Medicine, Stanford, California 94305
}

\begin{abstract}
Parasaggital knife cuts that transect the mediolateral connections between the medial and lateral preoptic areas and anterior and lateral hypothalamic structures severely disrupt maternal behavior in the postpartum lactating rat. These cuts increased stress-induced pituitary-adrenal responsiveness in lactating females while having no effect in virgins. These data support the hypothesis that pituitary-adrenal buffering that accompanies lactation facilitates the expression of maternal behavior.
\end{abstract}

The initiation of maternal behavior depends on endocrine changes that accompany pregnancy and parturition (Rosenblatt \& Siegel, 1975; Slotnick, Carpenter, \& Fusco, 1973). Once initiated, this caretaking behavior can proceed independent of hormonal influence (Obias, 1957) and is maintained through stimulation provided by the pups (Rosenblatt, 1969). While these behaviors are maintained by pup stimulation and proceed independent of hormonal control, there are a number of endocrine changes that normally accompany the expression of maternal behavior and are specific to lactation (Stern \& Levine, 1975).

One of the endocrine changes that accompanies lactation is the reduction (buffering) of pituitary-adrenal responsiveness to stressors (Stern, Goldman, \& Levine, 1973; Thoman, Wetzel, \& Levine, 1968). This buffering appears to be a consequence of the high titres of prolactin resulting from the suckling stimulation provided by the pups (Myers, Denenberg, Thoman, Holloway, \& Bowerman, 1975). Recently we have suggested that this pituitary-adrenal buffering facilitates the lactating female's responsiveness to changing patterns of stimuli provided by her pups (Smotherman, Wiener, Mendoza, \& Levine, 1976).

This research was supported by Research Grant NICH\&HD02881 from the National Institutes of Health to Seymour Levine, who is also supported by USPHS Research Scientist Award K5-MH-19936 from the National Institute of Mental Health (NIMH). William Smotherman was supported by Biosciences Training Grant MH-8304 from NIMH and John Hennessy was supported by a fellowship from Foundations' Fund for Research in Psychiatry. The authors wish to thank Martin Miner for his technical assistance in the construction of the encephalotome, Helen $\mathrm{Hu}$ for the determination of plasma concentrations of corticosterone, and Ann Bergfors for preparation of the brains for histological analysis.
While maternal behavior is resistant to disruption once established (Rosenblatt, 1969), Numan (1974) has shown that lesions of the medial preoptic area (MPOA) and knife cuts that sever medial preopticanterior hypothalamic fiber connections severely disrupt maternal behavior. Other studies (Avar \& Monos, 1969) have implicated the MPOA and its lateral connection with the expression of maternal behavior in the postpartum rat mother. The present study was undertaken to determine whether a relationship existed between the disruption of maternal behavior following MPOA knife cuts and responsiveness of the pituitary-adrenal system.

\section{MATERIALS AND METHODS}

\section{Subjects}

Subjects for this experiment were virgin female Sprague-Dawley rats purchased from Simonsen Laboratories (Gilroy, California). The animals were approximately 75 days of age at time of breeding. They were housed three per pan in metal pans that measured $28 \times 48 \times 15 \mathrm{~cm}$ in a colony room where temperature and humidity remained constant and lights were regulated on a $12 \mathrm{~h}$ on/12 h off cycle (lights on at 8:00 a.m.). One-half of the virgin females were used for breeding. Two Long-Evans males were introduced into the cage of these females, where they remained for 5 days. Virgin females not used for breeding served as controls. They were housed, fed, and maintained under the same conditions. After breeding, all females were individually housed in clear plastic cages measuring $28 \times 48 \times 24 \mathrm{~cm}$ and provided with paper strips for nesting material. Food and water were continually available via an external food source and water bottle. Within $24 \mathrm{~h}$ after delivery and at a parallel time for virgin females, mothers were assigned to either sham or operated (MPOA knife cut) conditions. This assignment resulted in four groups $(n=8$ per group): MPOA lactator, SHAM lactator, MPOA virgin, SHAM virgin. The day after delivery was considered postpartum. Day 1.

\section{Surgical Procedures}

The surgical procedures were carried out under Nembutal anesthesia on postpartum Day 5. The knife cuts were intended to duplicate those of Numan (1974), using an encephalotome 
described by Sclafani \& Grossman (1969). Briefly, the knife consisted of a 27-ga guide cannula which was curved at the end so that a $0.125 \mu \mathrm{m}$ steel wire could extend from the tip at an angle of approximately $90^{\circ}$. The tip of the guide cannula was at the following coordinates: $\mathrm{AP}=6.5, \mathrm{H}=0.0, \mathrm{~L}=1.3$ (De Groot, 1959). The bend at the tip was approximately $0.5 \mathrm{~mm}$ and the wire was extended 1.2-1.4 $\mathrm{mm}$ from the tip in a rostral direction. After extension, the wire curved downward about $0.5 \mathrm{~mm}$, such that the cut would begin at $\mathrm{H}=-0.5 \mathrm{~mm}$. The entire assembly was lowered $2.8 \mathrm{~mm}$ or to the base of the brain. This procedure was repeated on the contralateral side of the brain. Pilot data showed that positioning of the guide cannula without extension of the wire caused no postoperative effect on either maternal behavior or plasma level of corticosterone. For this reason, in this study the sham treatment consisted of Nembutal anesthesia, drilling of the skull, and suturing. The guide cannula was not lowered into the brain. Pups from lactating females were kept warm under a heat lamp until mothers had recovered fully from the surgery. Following the experiment, all experimental (MPOA) animals were sacrificed, the brains removed from the calvaria and stored in Formalin $(10 \%)$. The tissue was sliced in $5-\mu \mathrm{m}$ sections through the region of the cut and stained with thionin.

\section{Maternal Behavior Tests}

Forty-eight hours, after surgery, on Day 7 postpartum, all lactating females were observed and the presence or absence of a nest was determined. After this determination, mother and litter were removed from the colony room to an adjacent anteroom. Here the mother was removed from the home cage to a holding cage for $60 \mathrm{sec}$. During this time the pups were scattered about the home cage (away from the nest if one was present). Mothers were returned to the cage and the cage to the colony room. After $10 \mathrm{~min}$, a count was made to determine the number of pups that had been retrieved to the nest. Virgin females at this time were removed from the colony room, removed from their cage for $60 \mathrm{sec}$, returned to their cage, and then to the colony room.

\section{Blood Collection and Stress Procedures}

On Day 8 postpartum, all females were taken individually from the colony room and then blood was taken to determine basal levels. Under light ether anesthesia, $0.8 \mathrm{ml}$ of blood was collected in heparinized syringes ( $1.0 \mathrm{cc}$ tuberculin) by cardiac puncture. All samples were collected within $1 \mathrm{~min}$ after exposure to anesthesia. This method of sample collection allows for the determination of existing (e.g., basal) levels of plasma corticosterone (Davidson, Jones, \& Levine, 1968). After $20 \mathrm{~min}$, females were reanesthetized and a second blood sample collected. This second sample (stress sample) determined changes in corticosterone resulting from the initial exposure to ether, cardiac puncture, and blood loss. The blood was centrifuged at 2,000 rpm for $20 \mathrm{~min}$ and plasma extracted and frozen until assay. All maternal behavior observations and blood samples were taken between 10:00 a.m. and 11:00 a.m.

\section{Corticosterone Assay}

The plasma was assayed by the flurometric micro-method of Glick, von Redlich, and Levine (1964). The basal and stress levels of plasma corticosterone resulting from this assay were analyzed separately in 2 (surgical conditions: MPOA, SHAM) by 2 (parity: VIRGIN, LACTATOR) ANOVAs.

\section{RESULTS}

\section{Anatomical}

A schematic representation of the damage produced by the knife cuts is shown in Figure 1. As can be seen, the knife produced linear cuts through the
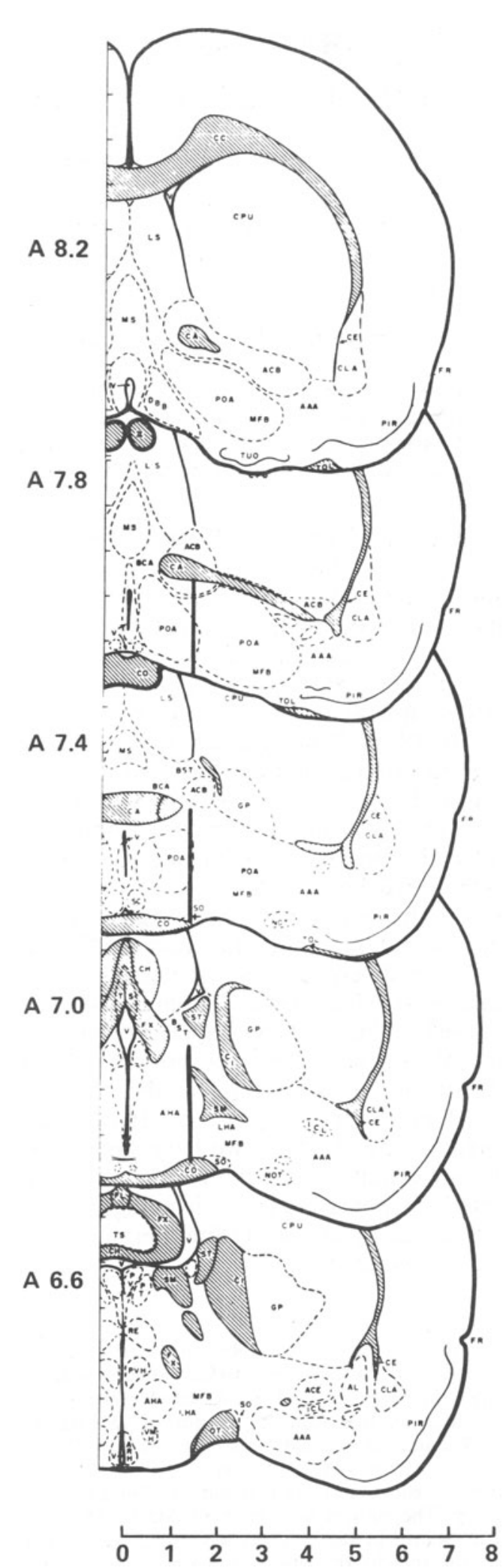

Figure 1. Schematic representation (after De Groot, 1959) of the extent of MPOA knife cuts. 
hypothalamus from a point just anterior to the preoptic area $(\mathrm{AP}=8.0-7.8)$ and extending to the rostral tip of the ventromedial and paraventricular nuclei (AP $=6.8-6.6)$ so as to sever all mediolateral connections. In the lateral dimension, the cuts were just medial to the supraoptic nucleus at the level of the preoptic areas and just lateral to the fornix columns more posteriorly $(\mathrm{L}=1.3-1.5)$. The dorsoventral extent of the cuts ran from the base of the brain to the level of the anterior commissure anteriorly and to the fornix columns in the vicinity of the anterior hypothalamic area. All cuts were reasonably bilaterally symmetric. No damage to the optic tract was observed, although some cuts ran into the caudal portions of the diagonal band of Broca rostrally.

Although the histological analysis was performed without knowledge of the results, no relationships could be discerned between variations in damage produced by the knife and those in maternal behavior or corticosterone measurement. Of importance here, all cuts separated the medial and lateral preoptic areas since it has recently been demonstrated (Conrad \& Pfaff, 1975) that most of the axonal projections between the medial preopticanterior hypothalamic continuum and the more lateral medial forebrain bundle arise from the medial preoptic area.

\section{Maternal Behavior}

Results of lactating females' maternal behavior are summarized in Table 1. Mothers with MPOA knife cuts built fewer nests than mothers that had undergone the sham operative procedure, $p<.01$ (Fishers exact probability test; Siegel, 1956, p. 96). In addition, MPOA mothers retrieved fewer pups compared to SHAM control mothers, $\mathrm{t}(14)=6.87$, $\mathrm{p}<.001$.

\section{Plasma Corticosterone}

Corticosterone data are shown in Figure 2. Analysis of the basal corticosterone data indicated that neither surgical condition nor parity affected basal secretion. Analysis of stress levels indicated that lactating females were less responsive than virgin females, $F(1,28)=50.61, p<.001$. Further, as the

Table 1

Postoperative Maternal Behavior

\begin{tabular}{lccc}
\hline & $\begin{array}{c}\text { MPOA SHAM } \\
(\mathrm{n}=8)\end{array}$ & $\begin{array}{c}\text { Fishers Exact } \\
(\mathrm{n}=8)\end{array}$ & Probability Test \\
\hline $\begin{array}{l}\text { Nests } \\
\text { (Number Constructed) }\end{array}$ & 1 & 8 & $\mathrm{p}<.01$ \\
$\begin{array}{l}\text { Pup Retrieval } \\
\text { (Mean Percent Retrieved) }\end{array}$ & 13.9 & 100 & $\mathrm{t}(14)=6.87, \mathrm{p}<.001$ \\
\hline
\end{tabular}

Note-One mother in the MPOA group retrieved all her 13 pups. Without her data, mothers in this group retrieved 3 of 76 pups (3.4\%).

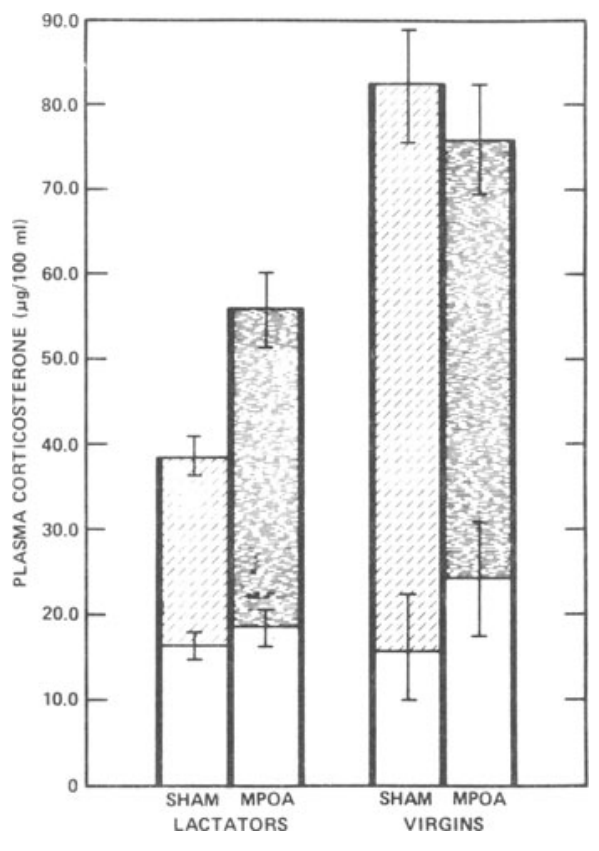

Figure 2. Mean basal (unshaded) and stress (shaded) levels of plasma corticosterone. Stress levels of corticosterone resulted from the initial exposure to ether, heart puncture, and blood loss. Vertical lines represent the standard error of the mean.

significant Surgical Condition by Parity interaction indicated, $\mathrm{F}(1,28)=6.17, \mathrm{p}<.01$, the MPOA fiber transections affected the pituitary-adrenal responsiveness of the lactating females, $F(1,28)=9.70$, $\mathrm{p}<.01$ (tests on simple main effects; Winer, 1971, p. 347).

\section{DISCUSSION}

Maternal behavior in the lactating female is severely disrupted following MPOA knife cuts. These data replicate the findings reported by Numan (1974) and confirm the importance of intact mediolateral connections of the medial preoptic area and the anterior hypothalamus for the expression of maternal behavior.

While the knife cuts did not affect basal corticosterone secretion in either virgin or lactator, responsiveness to an ether stress was changed. This change was seen only in lactating females, however, where the MPOA cuts resulted in higher plasma levels of corticosterone. This effect could be due to a reduction in the suckling-induced secretion of prolactin that would accompany the disruption of maternal behavior, since Myers et al. (1975) have shown that the intensity of suckling stimulation and stress-induced corticosterone release are inversely related.

Thoman et al. (1968) found that the lactating female still shows a buffered pituitary-adrenal re- 
sponse during lactation even after 4 days without pup stimulation. Not until $\mathbf{4 2}$ days postpartum (long after the cessation of suckling) do formerly lactating mothers show pituitary-adrenal responsiveness similar to that of virgin females. The Thoman et al. (1968) data, together with the finding of the present study (a reversal of this pituitary-adrenal buffering after only 2 days without pup stimulation), suggest that pituitary-adrenal changes that accompany lactation are indeed complex. The evidence correlating suckling-induced prolactin secretion with stress responsiveness of the pituitary-adrenal system (Myers et al., 1975) cannot fully account for this buffering effect.

It is an interesting possibility that the change in the response characteristics of the pituitary-adrenal system produced by the knife cuts is specific to the state of lactation. However, an alternative possibility exists. Ether stress could elicit maximum pituitaryadrenal responsiveness from both sham-operated and MPOA virgin females. This would then result in a ceiling effect that therefore masks the knife cut effect in MPOA virgin females.

We have reported elsewhere that while the lactating female normally shows reduced pituitary-adrenal responsiveness to stressors (e.g., ether stress), she remains responsive to stimuli from her pups (Smotherman et al., 1976). The degree of responsiveness is dependent on the intensity of treatment pups receive during a brief separation from the nest. More intense treatment of the pups (e.g., shock) during separation results in greater pituitary-adrenal responsiveness in mothers following reunion (Smotherman, Wiener, Mendoza, \& Levine, 1977). While virgin females show a pituitary-adrenal response to pups (Zarrow, Schlein, Denenberg, \& Cohen, 1972), this pattern of differential responsiveness is specific to the lactating female (Smotherman et al., 1976). The change in pituitary-adrenal responsiveness that accompanies MPOA knife cuts may help to explain the concomitant disruption of maternal behavior. It could be that mothers with MPOA knife cuts are more responsive to other classes of stimuli (stressors) and are, as a result, not responsive to stimuli produced by the pups. This lack of responsiveness would lead to the disruption of maternal behavior.

\section{REFERENCES}

Avar, Z., \& Monos, E. Biological role of lateral hypothalamic structures participating in the control of maternal behavior in the rat. Acta Physiologica Academiae Scientiarum Hungaricae, 1969, 35, 285-294.
Conrad, L. C. A., \& Pfaff, D. W. Axonal projections of medial preoptic and anterior hypothalamic neurons. Science, 1975, 190, 1112-1114.

Davidson, J. M., Jones, L. E., \& Levine, S. Feedback regulation of adrenocorticotropin secretion in 'basal' and 'stress' conditions: Acute and chronic effects of intrahypothalamic corticoid implantation. Endocrinology, 1968, 82, 655-663.

DE GROot, J. The rat forebrain in stereotaxic coordinates. Der Koninklijke Nederlandse Akademie van Wetenschappen, afd. Natuurkunde, 1959, 52, 1.40.

Glick, D., von Redlich, D., \& Levine, S. Fluorometric determination of corticosterone and cortisol in 0.02-0.05 milliliters of plasma or submilligram samples of adrenal tissue. Endocrinology, 1964. 74. 653-655.

Myers, M. M., Denenberg, V. H., Thoman, E., Holloway, W. R..\& Bowerman, D. R. The effects of litter size on plasma corticosterone and prolactin response to ether stress in the lactating rat. Neuroendocrinology, 1975, 19, 54-58.

Numan, M. Medial preoptic area and maternal behavior in the female rat. Journal of Comparative and Physiological Psychology, 1974, 87, 746-759.

Oв IAS, M. D. Maternal behavior of hypophysectomized gravid albino rats and the development and performance of their progeny. Journal of Comparative and Physiological Psychology, 1957. 50. 120-124.

RosenblatT, J. S. The development of maternal responsiveness in the rat. American Journal of Orthopsychiatry, 1969, 39, 36-56.

Rosenblatt, J. S., \& Siegel, H. I. Hysterectomy-induced maternal behavior during pregnancy in the rat. Journal of Comparative and Physiological Psychology, 1975, 89, 685-700.

Sclafani, A.. \& Grossman, S. P. Hyperphagia produced by knife cuts between the medial and lateral hypothalamus in the rat. Physiology and Behavior, 1969, 4, 533-537.

SIEGEL. S. Nonparametric statistics for the behavioral sciences. New York: McGraw-Hill. 1956.

Slotnick, B. M.. CARPENTER, M. L., \& Fusco, R. Initiation of maternal behavior in pregnant nulliparous rats. Hormones and Behavior, 1973, 4, 53-59.

Smotherman, W. P., Wiener, S. G., Mendoza, S. P., \& LEVINE, S. Pituitary-adrenal responsiveness of rat mothers to noxious stimuli and stimuli produced by pups. In K. Elliott \& D. W. Fitzsimons (Eds.), Ciba 45: Breast-feeding and the mother. Amsterdam: Elsevier, 1976.

Smotherman, W. P., Wiener, S. G., Mendoza, S. P., \& LEVINE, S. Maternal pituitary-adrenal responsiveness as a function of differential treatment of rat pups. Developmental Psychobiology, 1977, 10, 113-122.

Stern, J. M., \& Levine, S. Neuroendocrine changes during lactation in rats. Modern Problems in Paediatrics, 1975, 15, 106-116.

Stern, J. M., Goldman, L., \& Levine, S. Pituitary-adrenal responsiveness during lactation in rats. Neuroendocrinology, 1973, 12, 179-191.

Thoman, E. B., Wetzel, A., \& Levine, S. Lactation prevents disruption of temperature regulation and suppresses adrenocortical activity in rats. Communications in Behavioral Biology, 1968. Part A, 2, 165-171.

WINER, B. J. Statistical principles in experimental design (2nd ed.). New York: McGraw-Hill, 1971.

Zarrow, M. X., Schlein, P. A., Denenberg, V. H., \& Cohen, H. A. Sustained corticosterone release in lactating rats following olfactory stimulation from the pups. Endocrinology, 1972, 91. 191-196.

(Received for publication December 17, 1976; revision accepted February 4.1977.$)$ 